\title{
Competencia e identidad digital en redes sociales. Percepciones del profesorado en formación
}

\section{Digital Competence and Identity in Social Networks. Perceptions of Teachers in Training}

\author{
Julio Barroso-Osuna jbarroso@us.es — https://orcid.org/0000-0003-0139-9140 \\ Carmen Llorente-Cejudo karen@us.es — https://orcid.org/0000-0002-4281-928X \\ Antonio Palacios-Rodríguez aprodriguez@us.es — https://orcid.org/0000-0002-0689-6317 \\ Universidad de Sevilla, España
}

Fecha de recepción: 16 de abril de 2020

Fecha de aceptación: 1 de mayo de 2020

Fecha de publicación: 30 de junio de 2020

Favor de citar este artículo de la siguiente forma:

Barroso-Osuna, J., Llorente-Cejudo, C. y Palacios-Rodríguez,A. (2020).

Competencia e identidad digital. Percepciones del profesorado en formación.

AULA, Revista de Humanidades y Ciencias Sociales, 66 (2), 53-64.

DOI:https: //doi.org/10.33413/aulahcs.2020.66i2.139

\section{RESUMEN}

La identidad digital muestra todos los aspectos relativos a un usuario que está registrado en la Red, conscientemente o no; entre ellos, su competencia comunicativa y social, la cual queda claramente reflejada en las redes sociales, donde se manifiestan opiniones, relaciones e imágenes. El presente estudio parte de la necesidad de conocer cuáles son las percepciones del alumnado universitario en cuanto a su configuración de la identidad en línea y el desarrollo de la competencia digital. Para ello, se elabora un instrumento de corte cuantitativo que, una vez validado a través del juicio de expertos, se administra a un total de 252 estudiantes de la Facultad de Ciencias de la Educación de la Universidad de Sevilla. Entre los resultados más significativos, destacar que los alumnos usan de manera poco frecuente las redes sociales vinculadas al ámbito profesional. Preguntados sobre su competencia en la gestión de su identidad digital, la mayoría de los encuestados manifiestan tener «poca». Por este motivo, una de las conclusiones del estudio está dirigida hacia la necesidad de vertebrar planes de acción formativa desde la universidad, destinados al desarrollo profesional y educativo de las redes sociales.

Palabras clave: redes sociales, competencia digital, identidad digital, educación superior.

\begin{abstract}
Digital identity shows all the aspects related to a user who is registered on the network, consciously or not; among them, their communicative and social competence, which are clearly reflected in social networks, where opinions, relationships and images are manifested. This study starts from the need to know what are the perceptions of university students regarding their configuration
\end{abstract}


of online identity and development of digital competence. To do this, a quantitative instrument is developed that, once validated through the judgment of experts, is administered to a total of 252 students from the Faculty of Education Sciences of the University of Seville. Among the most significant results, it stands out that students infrequently use social networks linked to the professional field. When asked about their competence in managing their digital identity, the majority of those surveyed stated that they have «less» competence in protecting their digital identity. For this reason, one of the conclusions of the study is directed towards the need to structure training action plans from the university aimed at the professional and educational development of social networks.

Keywords: social networks, digital competence, digital identity, higher education.

\section{Las redes y la identidad digital en la formación del docente}

Si se hace un estudio en cualquiera de los niveles educativos por los que el estudiante transcurre a lo largo de su formación académica, no cabe duda que la competencia digital ha tomado un especial protagonismo en torno a los diferentes aspectos curriculares. Esto es debido a la gran demanda de la sociedad y del mercado laboral (Consejo de la Unión Europea, 2018; Gutiérrez-Castillo y Gómez-del-Castillo, 2015).

La Comisión Europea subraya que, la competencia digital es una de las competencias clave que cualquier joven debe haber desarrollado al finalizar la enseñanza obligatoria para poder incorporarse a la vida adulta de manera satisfactoria, y ser capaz de desarrollar un aprendizaje permanente a lo largo de la vida (Bonilla-del-Río y Aguaded, 2018; Llorente e Iglesias, 2018).

En diferentes trabajos se aborda esta temática, donde se hace referencia a que se debe superar la visión de la capacitación meramente instrumental para alcanzar otras dimensiones, tales como: la semiológica/estética, curricular, pragmática, psicológica, productora/diseñadora, seleccionadora/evaluadora, crítica, organizativa, actitudinal e investigadora (Cabero, 2004). Dichos autores también creen conveniente no dejar de lado algunos principios fundamentales en el desarrollo de la competencia digital de los estudiantes, como: el valor de la práctica y la reflexión sobre la misma, la participación del profesorado en su construcción y determinación, su diseño como producto no acabado, centrarse en medios disponibles para el profesorado, situarse dentro de estrategias de formación más amplias que el mero individualismo, alcanzar dimensiones más extensas como la planificación, diseño $\mathrm{y}$ evaluación, y fomentar la coproducción de materiales entre profesores y estudiantes.

Por su parte, la Comisión Europea (2013), desde la visión institucional, incide en que «la competencia digital es un prerrequisito para que los estudiantes de todas las edades puedan beneficiarse por completo de las nuevas posibilidades que ofrece la tecnología para un aprendizaje más eficaz, motivador e inclusivo» (p.19).

En el Espacio Europeo de Educación Superior (EEES), la realidad entra en conflicto con esta visión académica y con las postulaciones de la Comisión Europea sobre la competencia digital, todo ello debido a la pobre (en ocasiones, testimonial) presencia de dicha competencia en los planes de estudio, especialmente en los relacionados con el campo de la educción y en la formación del profesor universitario (Cabero-Almenara, Barroso-Osuna, Palacios-Rodríguez, y Llorente-Cejudo, 2020). Al revisar con detenimiento dichos planes de estudio, no solo la competencia digital es escasa, sino que también escasean los objetivos y las materias en las que se desarrollan los contenidos propios de la temática (Aguilar Gavira, 2015). Es relevante incidir en que existen planes de estudio en España de formación del profesorado y de pedagogía, carentes de materia alguna que promueva de manera específica dicha competencia. Esta situación es comentada por Gutiérrez, Palacios y Torrego 
(2010), los cuales señalan que incluso los usuarios habituales de tecnologías ignoran su potencial didáctico y las posibles formas de integración en los currículos de la enseñanza obligatoria. De todo esto, se extrae que la posibilidad de considerar las TIC como medios de expresión creativa y/o de participación democrática, queda lejos de las percepciones de los discentes universitarios.

Así pues, se genera la necesidad de definir cuáles son los elementos curriculares que conforman la formación necesaria para la adquisición de la competencia digital, en cualquier etapa educativa y, por ende, se requiere crear un marco común con amplio reconocimiento y consenso (Porlán, 2017). Es la propuesta generada por el Joint Research Centre de la Comisión Europea (Carretero, Vuorikari, y Punie, 2017; Redecker y Punie, 2017) que, con los resultados del proyecto DigComp ha publicado un marco sólido sobre el cual elaborar un currículo en el campo digital (competencias, objetivos, estándares, etc.). Sobre este último se han basado diferentes instituciones para generar los actuales marcos de competencias digitales docentes (Cabero-Almenara y Palacios-Rodríguez, 2020; INTEF, 2017; Redecker y Punie, 2017).

En este amplio campo de actuación, se hace imprescindible que los estudiantes y futuros docentes estén capacitados para movilizar y utilizar los recursos tecnológicos que tienen a su disposición, incorporando a su vez la diversidad de signos que utilizan (Alonso-García, Morte-Toboso, y Almansa-Núñez, 2014; Peña Hita, Rueda López, y Del Carmen Pegalajar Palomino, 2018). Ello requiere, fundamentalmente, un nuevo tipo de alfabetización digital, que se centre no solo en los medios impresos y los códigos verbales en los cuales se sustentan en su día a día; sino también en la diversidad de los multimedia que existen en la actualidad (Dans Álvarez de Sotomayor y Muñoz Carril, 2016; Díaz y Martín Jaime, 2015). Cabero et al. (2018) inciden en la necesidad de reclamar la falta de capacitación de los estudiantes en una competencia digital que les lleve a evaluar y discriminar, la pertinencia de la información existente en Internet y en los recursos abiertos que por ella circulan. Esta capacitación de los docentes se puede plantear desde nuevas perspectivas (CEJAS et al. 2016). Se resalta la importancia de la transformación del rol docente como persona que ha de encargarse de diseñar situaciones significativas de aprendizaje, por lo que no hay duda de que adquirir y desarrollar con adecuación las diferentes competencias digitales $y$, en concreto, las que afectan a nuestra identidad digital, es un reto que el alumnado universitario de Educación debe alcanzar (Sáez y Sancho, 2017). Es decir, los futuros docentes, han de lograrlo tanto para su beneficio personal, como para el beneficio de sus alumnos. Uno de los aspectos más preocupantes a tener en cuenta para los estudiantes de Educación en la actualidad, es tener presente el concepto sobre la identidad digital (Sampedro Requena, 2015).

\section{La repercusión de las redes \\ y la identidad digital}

La digitalización de la sociedad confiere a cada persona una identidad digital que crece y se desarrolla paralela a la identidad del mundo físico o real, generándose procesos relativos al manejo de información personal cada vez más intensivos, exigentes y automatizados que controlan nuestras vidas.

La identidad digital hace referencia a toda aquella información resultante de la indagación sobre nosotros en los buscadores de Internet (nombre completo, documento de identidad, teléfono, email, etc.). En ocasiones, esta información puede estar presente en Internet de forma poco consciente para nosotros $\mathrm{u}$ otras personas ajenas, que pueden manipular o etiquetar dicha información (amigos, desconocidos, Administración Pública, etc.), de tal modo que se puede crear una identidad digital mínima sin ser conscientes ni haberlo autorizado.

Castañeda y Camacho (2012) definen la identidad digital como aquellos aspectos de la tecnología digital que median en la experiencia de la identidad construida por las personas y también condicionada por los factores sociales. 
Una vez situados en la comprensión y la importancia de la competencia digital y la identidad digital, se procede a analizar la incidencia de ésta en las personas y, concretamente, en la necesidad de promover una repercusión positiva en el docente (Sánchez-Caballé, Gisbert-Cervera, y Esteve-Mon, 2019; Sánchez y Porlán, 2018).

Camacho (2015) afirma la intrusión de nuevas tecnologías sin tener como objetivo la interactividad o intervención del usuario, siendo necesaria la formación sólida del usuario sobre la Web 3.0 y las repercusiones de la misma, relacionándose esto con la teoría de una perspectiva instrumental que no considerará la repercusión social de la interacción del usuario en la red. Por ende, la identidad digital muestra todos los aspectos relativos a un usuario que está registrado en la red conscientemente o no; entre ellos, su competencia comunicativa y social. Además, esta queda reflejada muy claramente en las redes sociales, donde se pueden observar las opiniones, relaciones e imágenes del docente (Díaz-Lázaro, Solano Fernández, y Vera, 2017; Gómez García, Ruiz Palmero, y Sánchez Rodríguez, 2014; López de la Madrid, Flores Guerrero, y Espinoza de los Monteros Cárdenas, 2015). Conjuntamente, en la identidad digital, también quedan expresados los valores, la moral y la ética de un usuario (Marín Díaz, 2013). Esto puede tener repercusiones, tanto positivas como negativas. Es necesaria la tenencia de un mínimo de ética en la red para poder usarla con buenos fines. No obstante, en el caso de la moralidad o los valores, pueden ser usados negativamente. Un ejemplo de ello, sería el rechazo de un candidato a una oferta de empleo porque aparezca en su identidad digital que tiene ideales afines a un partido político contrario al de la empresa que hace la oferta. Este ejemplo sería un mal uso de la identidad digital. Asimismo, las referencias personales y opiniones realizadas por familiares y amigos en la identidad digital influyen en el mundo personal y en la confianza que la persona proyecta en la Red (Mayor Buzón, García Pérez, y
Rebollo Catalán, 2019; Mena Guacas y Santoveña Casal, 2018). Muchos comentarios positivos por parte de amigos y familia hacen que el resto vea a esta persona confiable y triunfadora, logrando que triunfe en otras relaciones más lejanas. Por último, cabe destacar que todos los datos y archivos que son compartidos y que conforman la identidad digital, son cedidos por el propio usuario de forma voluntaria y legal a los perfiles que componen su identidad digital y a las compañías que los gestionan; de tal forma que es muy fácil no saber para qué se están usando estos datos, además de ser muy difícil el poder eliminarlos (Monreal Guerrero, Parejo Llanos, y Cortón de las Heras, 2017). Por lo tanto, tenemos que ser prudentes a la hora de subir a la Red información sobre nosotros, pues nuestra identidad digital es muy importante.

La carencia y escasez de estudios e investigaciones que ofrezcan resultados y conclusiones a la posible relación entre el uso de las redes sociales y su vinculación con la identidad digital, fue el detonante a partir del cual se considera la necesidad de realizar el presente estudio de investigación, cuyos objetivos generales a alcanzar se pueden declarar en los siguientes términos:

-Analizar la percepción del alumnado universitario de la Facultad de Ciencias de la Educación de la Universidad de Sevilla, sobre las redes sociales digitales y su relación con la identidad digital.

-Diseñar y validar un instrumento para el análisis de las percepciones del alumnado universitario sobre sus competencias digitales, respecto al uso de las redes sociales y su identidad digital, a través del juicio de expertos.

-Identificar y proponer acciones de mejora en la formación de los alumnos universitarios sobre sus competencias digitales, respecto al uso de las redes sociales y la identidad digital.

\section{Metodología}

El estudio desarrollado se lleva a cabo a través de una metodología de corte cuantitativo, mediante 
la cual los resultados se obtienen del registro que los alumnos realizan usando un cuestionario disponible online (https://bit.ly/3bdGbP1).

De acuerdo con Bisquerra (2004) y Arnal et al. (1992), el estudio que se presenta se puede enmarcar en el tipo descriptivo. Concretamente, en los denominados «ex post facto», aquellos en los que el investigador se plantea la validación de las hipótesis cuando el fenómeno ya ha sucedido o se encuentra en fase de desarrollo.

Dicho instrumento está compuesto por seis grandes categorías o dimensiones:

a. Datos personales;

b. Redes sociales, uso y actualización;

c. Tipos de uso de las redes sociales por el alumnado;

d. Repercusión de la identidad digital del alumnado en las redes sociales;

e. Percepción de las competencias digitales del alumnado vinculadas a su identidad digital y las redes sociales que la componen; y

f. Formación en competencia digital sobre redes sociales e identidad digital. En su totalidad, el cuestionario está compuesto por 45 ítems, distribuidos en las dimensiones expuestas con anterioridad.

El muestreo realizado fue de tipo intencional. Los datos que se han obtenido responden al deseo de participar de los estudiantes que cumplimentaron el cuestionario, y a la facilidad de acceso al instrumento. El número total de participantes fue de 252 estudiantes de la Facultad de Ciencias de la Educación de la Universidad de Sevilla, en su mayoría de género femenino (76,6\%), de edades comprendidas entre los 18 y los 29 años, mayoritariamente cursando la Licenciatura en Educación Primaria (76,2\%), con dedicación completa a sus estudios $(91,3 \%)$, y procedente de los estudios de bachillerato $(70,2 \%)$ o de un grado superior de FP $(25,0 \%)$. Todos los participantes están cursando alguno de los grados que ofrece la Facultad de Ciencias de la Educación de la Universidad de Sevilla: Pedagogía, Educación Primaria, Educación Infantil y de la Actividad Física y del Deporte.
Como método de validación, se emplea el juicio de expertos, donde se solicita a una serie de sujetos la demanda de un juicio hacia el instrumento. Para ello, se procede al envío del instrumento a un total de 30 profesores vinculados al ámbito de la Tecnología Educativa y las Tecnologías de la Información y la Comunicación en Educación de diferentes universidades (Universidad de Sevilla, Universidad de Huelva, Universidad de Baleares, Universidad de Córdoba y Universidad del País Vasco), obteniendo diferentes modificaciones y sugerencias que se incorporan a la versión final del instrumento y, de la cual, se obtiene su versión definitiva. Para la realización de los análisis estadísticos se empleó el programa de análisis estadístico SPSS.

La obtención del índice de fiabilidad del instrumento se logra mediante el coeficiente Alfa de Cronbach, tal y como sugieren O'Dwyer y Bernauer (2014) para este tipo de instrumentos. Los valores obtenidos para la globalidad del instrumento, y para cada una de las dimensiones que lo conforman, se presentan en la tabla 1.

Estos valores, de acuerdo con diferentes autores (Mateo, 2004; O'Dwyer y Bernauer, 2014), pueden considerarse de altos o muy altos y denotan un nivel aceptado de fiabilidad del instrumento.

\begin{tabular}{|cc}
\hline ENFOQUE & VALOR ALFA \\
\hline Total instrumento & 0,951 \\
\hline Redes sociales, uso y actualización & 0,893 \\
\hline Tipos de uso de las redes sociales & 0,900 \\
\hline Repercusión de la ID & 0,882 \\
\hline Percepción de las CD & 0,800 \\
\hline Formación en CD & 0,900
\end{tabular}

Tabla 1. Alfa de Cronbach para el instrumento elaborado.

\section{Resultados}

En la figura 1 se incluyen los datos obtenidos tras peguntar a los encuestados por las redes sociales y la frecuencia quincenal de uso y actualización. 


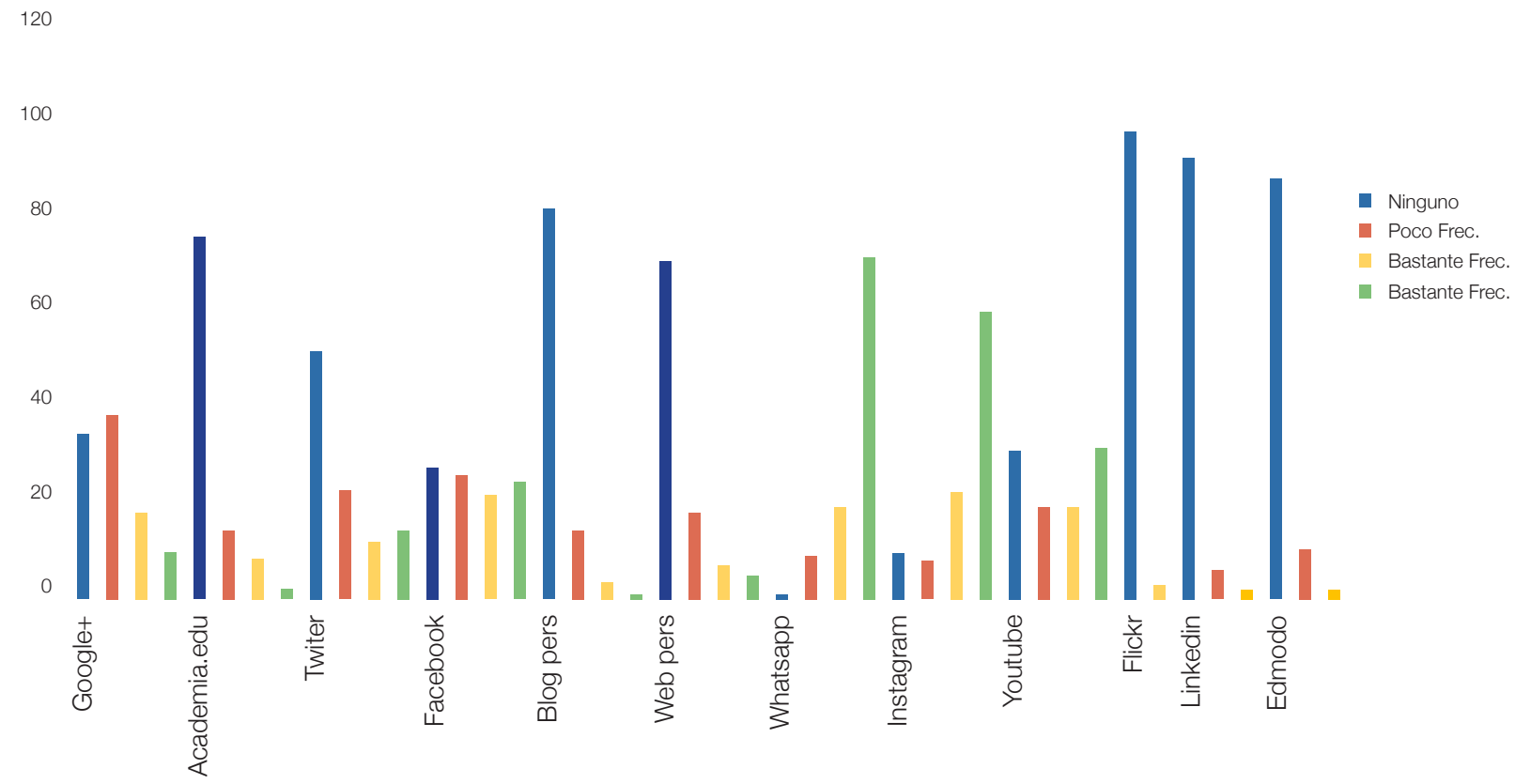

Figura 1. Frecuencia uso/actualización de redes sociales.

Como se puede observar en la representación gráfica, las redes sociales más usadas/ actualizadas por los encuestados y, por ende, en la que mantienen una mayor presencia son WhatsApp, en la que el 70,6\% de los encuestados manifiesta realizar un uso/actualización «muy frecuente», seguido de Instagram para el que el 59,9\% hace un uso/actualización «muy frecuente»y un $22,2 \%$ que indica que hace un uso «bastante frecuente». En el caso de la red social Youtube, se puede ver cómo existe cierto equilibrio entre los encuestados que manifiestan hacer un uso «bastante frecuente» y «muy frecuente» $(18,7 \%$ y $31,3 \%$ respectivamente) y los que manifiestan realizar un uso «poco frecuente» o «ninguno» (19,0\% y 31,0\% respectivamente). Algo parecido ocurre con la red social Facebook, aunque en este caso, son más los encuestados que manifiestan hacer un uso poco frecuente o ningún uso de esta red social $(25,8 \%$ y $27,8 \%$ respectivamente) frente a los que nos indican que hacen un uso bastante frecuente o muy frecuente de esta red social $(21,8 \%$ y $24,6 \%$ respectivamente). En el caso de Twitter, solo el $14,3 \%$ de los encuestados manifiesta hacer un uso/actualización «muy frecuente», el $11,9 \%$ «bastante frecuente», frente al 51,6\% indica que no hace ningún uso/actualización y el $22,2 \%$ la usa de una manera «poco frecuente». A la luz de los datos representados, el resto de las redes sociales (Google+, Academia.edu, Blog personal, Web personal, Flickr, Linkedln y Edmodo) por las que se les pregunta a los encuestados, no son usadas de manera significativa por estos.

A continuación, se procede a comentar el uso que los encuestados hacen de las redes sociales en las que participan. Para ello, y para hacer más clara la exposición de los resultados, se han sumado los porcentajes de las opciones «nada» y «poco» uso, y los porcentajes de los que manifiestan hacer «bastante» y «mucho» uso de estas. En esta línea se puede decir que los alumnos encuestados utilizan las redes sociales para: «mejorar e implementar su formación en otros ámbitos no profesionales $(40,8 \%)$, «búsqueda de empleo» $(32,5 \%)$, «mejorar e implementar su competencia profesional» $(31,8 \%)$, «opinar 
sobre temas diversos» $(30,2 \%)$, «mejorar e implementar su competencia investigadora» $(22,3 \%)$, «incorporarse a redes docentes» (20,3\%), «compartir producción de divulgación docente» $(19,1 \%)$, «buscar o incorporarse a redes de innovación» $(17,1 \%)$, «compartir producción científica» $(14,7 \%)$ y por último «incorporarse a redes de investigación» $(12,3 \%)$.

En el siguiente bloque del cuestionario se pretende recoger información en relación al conocimiento que tienen los encuestados con respecto a las repercusiones que pueden llegar a tener las redes sociales y la configuración de una identidad digital. Para ello se les preguntó sobre los siguientes aspectos:

-Identidad digital y representación a nivel personal: el 83,3\% de los encuestados manifiesta ser consciente de este aspecto, frente al $16,7 \%$ que declara no ser consciente.

-Identidad digital y representación a nivel profesional: el $72,3 \%$ de los encuestados manifiesta ser consciente frente al $27,7 \%$ que dice no ser consciente.

-Identidad digital como reflejo de sus valores, moral y su ética en la red: el $80,9 \%$ es consciente, frente al 19,1\% que lo desconoce.

-Identidad digital como medio relevante para ofrecer información para los empleadores: el $71,8 \%$ es consciente, frente al $28,2 \%$ que 10 desconoce.

-Identidad digital como muestra de su competencia comunicativa y social: el $80,1 \%$ declara ser consciente, frente al 19,9\% que lo desconoce.

-El buen desarrollo de una identidad digital es de gran relevancia para los usuarios: el $78,2 \%$ de los encuestados es consciente, frente al $21,8 \%$ que dice desconocerlo.

-Conocimiento de que en una misma plataforma/red social puede tener más de un perfil digital para diferentes usos y con diferentes niveles de privacidad: el 78,2\% de los encuestados es consciente, frente al $21,8 \%$ que lo desconoce.
-Las referencias personales y opiniones sobre el usuario que realizan amigos o familiares en su identidad digital y perfiles, influyen en su mundo laboral y en la confianza proyectada por este usuario en la red: el $81,3 \%$ de los usuarios es consciente de este aspecto frente al 18,7\% que desconoce esta posibilidad.

-Conocimiento en relación a la mala práctica de compartir archivos privados en los perfiles de sus redes: el 90,9\% es consciente de la imprudencia de compartir archivos privados en los perfiles de sus redes, frente al 9,1\% que lo desconoce.

-Conocimiento sobre que la cesión de datos y archivos compartidos (y con los que trabaja en sus redes sociales) son cedidos de forma voluntaria y legal a las compañías que los gestionan: el 78,8\% de los encuestados es consciente de este aspecto, frente al 21,2\% que lo desconoce.

Cuando se pregunta a los encuestados en relación a su competencia en la gestión de su identidad digital, el 39,3\% manifiesta que tenía «bastante» conocimiento en estos menesteres y el $11,1 \%$ considera que tiene «mucha» competencia a la hora de proteger su identidad digital; pero, por otro lado, se señala que el 40,9\% de los encuestados manifiesta tener «poca» competencia a la hora de proteger su identidad digital y el 8,7\% manifiesta no tener «nada» de esa competencia. Algo similar ocurre cuando se le preguntaba a los encuestados sobre la competencia que presentan en relación a las acciones encaminadas a la protección de sus datos personales. El $43,3 \%$ manifiesta tener «bastante» competencia, el 22,2\% «mucha» competencia y por el contrario el 28,6\% de los encuestados manifiesta tener «poca» competencia y el $6 \%$ «nada» de competencia en todas aquellas acciones encaminadas a proteger su identidad digital.

En la figura 2 se incluye la representación gráfica de los resultados obtenidos al preguntar a los encuestados sobre donde el tipo de formación adquirida en redes sociales e identidad digital. 



\#ada $\quad$ Poco $\square$ Bastante $\square$ Mucho

Informal

Figura 2. Formación adquirida en redes sociales e identidad digital.

Como se puede observar, los encuestados tienden a formarse de manera informal («Bastante» 45,6\%, «Mucho»19,4\%). Es decir, con amigos, familiares, compañeros, medios de comunicación, Internet, etc. Las otras modalidades, la formal, acciones formativas promovidas desde universidades («Bastante» 25\%, «Mucho» $2,4 \%$ ) y la no formal, a través de acciones formativas promovidas por empresas, sindicatos, servicios de empleo... («Bastante» 19\%, «Mucho» 2\%), no son elegidas por los encuestados como medio para formarse en redes sociales e identidad digital.

Por último, se pregunta a los encuestados sobre si demandan más formación sobre la gestión de su identidad digital y redes sociales y sobre la protección de datos personales y redes sociales. En los dos casos, los encuestados manifiestan necesitar más formación en la gestión de su identidad digital (64.6\%) y redes sociales, y manifiestan necesitar formación que les ayude a proteger sus datos personales y redes sociales $(69.9 \%)$.

\section{Conclusiones}

En primer lugar, se ha podido comprobar la validez y fiabilidad de un instrumento propio, cuyo eje fundamental son las competencias digitales de los estudiantes y su identidad digital. Un tema que está en auge en lo que respecta al ámbito de las Tecnologías de la Información y la Comunicación (TIC), sobre todo en lo que a los niveles de educación superior se refiere (Ángeles Peña Hita et al., 2018; Sánchez-Caballé et al., 2019; Sánchez y Porlán, 2018). Resulta relevante, no solo por la temática, si no por la escasez de instrumentos que desde la perspectiva de la identidad digital y su vinculación con un tema tan en boga como las redes sociales, existen en el panorama de la investigación educativa en España.

Con respecto a los datos obtenidos debe destacarse que, de manera general, se puede afirmar que los estudiantes de la Facultad de Educación de la Universidad de Sevilla usan y actualizan de manera poco frecuente las redes sociales en su identidad digital en lo que respecta 
a sus propias competencias digitales profesionalizantes o académicas (Linkedin, Academia. edu, etc.); sin embargo, es notorio que éstos sí las usan, actualizan y mantienen al día con respecto a su identidad digital, cuando se refiere a redes sociales con carácter de ocio (trátense algunas de las más conocidas y utilizadas como WhatsApp, Instagram o Facebook). Estos resultados coinciden con los encontrados en otros estudios e investigaciones vinculadas con las actitudes que los alumnos muestran hacia las TIC y las redes sociales (Sampedro y Marín, 2015; Cabero et al., 2016), donde apuntan que tienen una actitud significativa hacia las mismas, pero señalan que los alumnos no son tan «nativos» en su dominio como desde ciertos sectores se ha hecho creer.

Otros estudios apuntan cómo los alumnos muestran que se encuentran capacitados en el uso de las redes sociales, uso de sitios para compartir vídeos en la red, o referidos a la publicación de fotografías. En contrapartida, no presentan una fuerte formación en otras TIC como los blog, las wikis, las videoconferencias o los marcadores sociales (Cabero-Almenara, Arancibia Muñoz, Valdivia Zamorano y Araneda Riveros, 2018; Monreal Guerrero et al., 2017; Sáez y Sancho, 2017). Así mismo, coinciden en que las redes que los alumnos principalmente utilizan son Facebook y Twitter, lo que permite apuntar que las experiencias que en los últimos tiempos se están llevando a cabo para su incorporación en el terreno educativo (Duffy, 2011; Llorens y Capdeferro, 2011; Túñez y García, 2012; WebLong y Chun-Yi, 2013), cuentan con la ventaja inicial del conocimiento que los alumnos tienen respecto a las mismas.

Todo ello nos lleva a poder establecer una de las conclusiones a las que se puede llegar a través del presente estudio: que los estudiantes de niveles de educación superior necesitan de planes de acción formativa destinados al uso educativo de las redes sociales vinculadas con la significatividad de su identidad digital. En otras palabras, la institución educativa universitaria debe fomentar el uso educativo y profesionalizante de las redes sociales para los estudiantes, un campo con líneas emergentes en el contexto investigador (Gómez García et al., 2014).

Por otro lado, el estudio ha permitido identificar una serie de necesidades en lo que respecta a la importancia que los usuarios de las diferentes redes sociales otorgan a procesos como la búsqueda de trabajo o la incorporación en su ámbito profesional. En este sentido, consideramos que el plan de acción formativo al que hacíamos alusión líneas más arriba, debe contemplar esta necesidad como prioritaria, teniendo en cuenta que está vinculada a su desarrollo profesional en el campo educativo correspondiente; ya que en la mayoría de las ocasiones, los encuestados indicaron un alto grado de desconocimiento sobre la relevancia que estás poseen en diferentes ámbitos, tanto personales como profesionales.

\section{Referencias}

Aguilar Gavira, S. (2015). Cabero, J. (dir.) (2014). La formación del profesorado en TIC: Modelo TPACK (Conocimiento Tecnológico, Pedagógico y de Contenido). Sevilla: Secretariado de Recursos Audiovisuales y Nuevas Tecnologías de la Universidad de Sevilla. [Reseñas]. Píxel-Bit, Revista de Medios y Educación, 46, 253-254. https://doi.org/10.12795/ PIXELBIT

Alonso-García, S., Morte-Toboso, E., y Almansa-Núñez, S. (2014). Redes sociales aplicadas a la educación: EDMODO.

EDMETIC, 4(2), 88. https://doi.org/10.21071/edmetic.v4i2.3964

Ángeles Peña Hita, M., Rueda López, E. y Del Carmen Pegalajar Palomino, M. (2018). Posibilities of teaching networks in skills development higher education: Students's perceptions. Pixel-Bit, Revista de Medios y Educación, 53, 239-252. https://doi.org/10.12795/ pixelbit.2018.i53.16

Arnal, J.; Del Rincón, D. y Latorre, A. (1992). Investigación educativa. Fundamentos y metodología. Barcelona: Labor. 
COMPETENCIA E IDENTIDAD DIGITAL EN REDES SOCIALES. PERCEPCIONES DEL PROFESORADO EN FORMACIÓN

Bisquerra, R. (2004). Metodología de la investigación educativa. Madrid: La Muralla.

Bonilla-del-Río, M. y Aguaded, I. (2018). La escuela en la era digital: smartphones, apps y programación en Educación Primaria y su repercusión en la competencia mediática del alumnado. Píxel-Bit. Revista de Medios y Educación, 53, 151-163. https://doi.org/10.12795/ PIXELBIT.2018.I53.10

Cabero, J. (2004). Las TIC en la Universidad. Sevilla: MAD.

Cabero-Almenara, J., Arancibia Muñoz, M.L., Valdivia Zamorano, I. y Araneda Riveros, S.M. (2018). Percepciones de profesores y estudiantes de la formación virtual y de las herramientas en ellas utilizadas. Diálogo Educativo, 18(56), 149-163. http://dx.doi.org/10.7213/1981-416X.18.056.DS07

Cabero-Almenara, J. y Palacios-Rodríguez, A. (2020). Marco Europeo de Competencia Digital Docente «DigCompEdu» y cuestionario «DigCompEdu Check-In». EDMETIC. Revista de Educación Mediática y TIC, 9(1), 213-234. https://doi.org/10.21071/edmetic.v9i1.12462

Cabero-Almenara, Julio, Barroso-Osuna, J., Palacios-Rodríguez, A. y Llorente-Cejudo, C. (2020). Marcos de Competencias Digitales para docentes universitarios: su evaluación a través del coeficiente competencia experta. Revista Electrónica Interuniversitaria de Formación Del Profesorado, 23(2). https://doi.org/10.6018/ REIFOP.413601

Carretero, S., Vuorikari, R. y Punie, Y. (2017). DigComp 2.1: The Digital Competence Framework for Citizens With eight proficiency levels and examples of use. Luxembourg: Publication Office of the European Union. https://doi.org/10.2760/38842

Castañeda, L. y Camacho, M. (2012). Desvelando nuestra identidad digital. El profesional de la información, 21(4), 354-360. http://dx.doi.org/10.3145/epi.2012.jul. 04

Comisión Europea (2013). Education and Training Monitor 2013. Recuperado de http://ec.europa.eu/education/lifelong $\square$ learning $\square$ policy/progress_en.htm

Consejo de la Unión Europea. (2018). Recomendación del Consejo, de 22 de mayo de 2018, relativa a las competencias clave para el aprendizaje permanente. Bruselas: Diario Oficial de la Unión Europea.

Dans Álvarez de Sotomayor, I.y Muñoz Carril, P.C. (2016). Las redes sociales como motivación para el aprendizaje: opinión de los adolescentes. Innoeduca. International Journal of Technology and Educational Innovation, 2(1), 20. https://doi.org/10.20548/innoeduca.2016.v2i1.1041

Díaz, M.D.y Martín Jaime, J.J. (2015). Evaluación del perfil competencial de alumnos universitarios respecto a la
AULA, Revista de Humanidades y Ciencias Sociales

educación tecnológica. Innoeduca. International Journal of Technology and Educational Innovation, 1(1), 17. https://doi.org/10.20548/innoeduca.2015.v1i1.29

Díaz-Lázaro, J.J., Solano Fernández, I.M. y Vera, M. del M.S. (2017). Social learning analytics in higher education. An experienceat the primary education stage. Journal of New Approaches in Educational Research, 6(2), 119-126. https://doi.org/10.7821/naer.2017.7.232

Duffy, P. (2011). Facebook or faceblock: cautionary tales exploring the rise of social networking, in C. McLoughlin, (comps.), Web 2.0. Based e-learning, New York: Information Science Reference.

Gómez García, M., Ruiz Palmero, J. y Sánchez Rodríguez, J. (2014). Aprendizaje social en red. Las redes digitales en la formación universitaria. EDMETIC, 4(2), 71. https://doi.org/10.21071/edmetic.v4i2.3963

Gutiérrez, A., Palacios, A. y Torrego, L. (2010). La formación de los futuros maestros y la integración de las TIC en la educación: anatomía de un desencuentro. Revista de Educación, 3(52), 12-24.

Gutiérrez-Castillo, J-J. y Gómez-del-Castillo, M. (2015). Influencia de las TIC en los procesos de aprendizaje y comunicación de los estudiantes de educación. Revista de Pedagogía, 36(98), 34-51.

INTEF. (2017). Marco Común de Competencia Digital Docente. Madrid: Instituto Nacional de Tecnologías Educativas y Formación del Profesorado.

Llorens, F. y Capdeferro, N. (2011). Posibilidades de la plataforma Facebook para el aprendizaje colaborativo en línea. RUSC, Revista de Universidad y Sociedad del Conocimiento, 8(2), 31-45.

Llorente, P.A. e Iglesias, E.C. (2018). Desarrollo de la competencia digital en la formación inicial del profesorado de Educación Infantil. Píxel-Bit. Revista de Medios y Educación, 52, 97-110. https://doi.org/10.12795/PIXELBIT.2018.I52.07

López de la Madrid, M.C., Flores Guerrero, K. y Espinoza de los Monteros Cárdenas, A. (2015). Diversidad de usos de Facebook en la educación superior. Análisis desde un caso de estudio. Innoeduca. International Journal of Technology and Educational Innovation, 1(2), 106. https://doi.org/10.20548/innoeduca.2015.v1i2.1040

Marín Díaz, V. (2013). Los entornos personales de aprendizaje en el espacio formativo. EDMETIC, 2(1), 1. https://doi.org/10.21071/edmetic.v2i1.2856

Mateo, J. (2004). La investigación ex-post-facto. En R. Bisquerra (coord.), Metodología de la investigación educativa (pp. 195-230). Madrid: La Muralla.

Mayor Buzón, V., García Pérez, R. y Rebollo Catalán, Á. (2019). Explorando factores predictores de la competencia digital 
en las redes sociales virtuales. Pixel-Bit, Revista de Medios y Educación, 56, 51-69. https://doi.org/10.12795/ pixelbit.2019.i56.03

Mena Guacas, A. y Santoveña Casal, S.M. (2018). Whatsapp and Formulating Guidelines on Network Interaction during the Learning Process in the University Classroom. IJERI: International Journal of Educational Research and Innovation, 0(11), 121-136.

Monreal Guerrero, I.M., Parejo Llanos, J.L. y Cortón de las Heras, M.D. la O. (2017). Alfabetización mediática y cultura de la participación: retos de la ciudadanía digital en la Sociedad de la Información. EDMETIC, 6(2), 148. https://doi.org/10.21071/edmetic.v6i2.6943

O'Dwyer, L. y Bernauer, J. (2014). Quantitative research for the qualitative researcher. California: Sage.

OCDE (2018). Desigualdad de ingresos: La brecha entre ricos y pobres. Recuperado de http://www.oecd.org/economy/desigualdad-de-ingresos-9789264300521-es. htm

Porlán, I.G. (2017). Identidad digital, ser, estar y actuar en la red. Revista Interuniversitaria de Investigación En Tecnología Educativa, 3(2), 25-33. https://doi. org/10.6018/RIITE/2017/297721

Redecker, C. y Punie, Y. (2017). Digital Competence of Educators DigCompEdu. Luxembourg: Publications Office of the European Union.

Sáez, I.A. y Sancho, N.B. (2017). The integrated curriculum, university teacher identity and teaching culture: The effects of an interdisciplinary activity. Journal of New
Approaches in Educational Research, 6(2), 127-134. https://doi.org/10.7821/naer.2017.7.235

Sampedro Requena, B.E. (2015). Las TIC y la educación social en el siglo XXI. EDMETIC, 5(1), 8. https://doi. org/10.21071/edmetic.v5i1.4014

Sampedro, B. y Marín, V. (2015). Conocimiento de los futuros educadores sociales de las herramientas web 2.0. Pixel-Bit. Revista de Medios y Educación, 47, 211-222. https://doi.org/10.12795/pixelbit.2015.i47.03

Sánchez, F.L.S. y Porlán, I.G. (2018). La Identidad Digital del alumnado universitario: Estudio descriptivo en la facultad de Educación de la Universidad de Murcia. Revista Interuniversitaria de Investigación En Tecnología Educativa, 4, 32-46. https://doi.org/10.6018/RIITE/2018/30001

Sánchez-Caballé, A., Gisbert-Cervera, M. y Esteve-Mon, F. (2019). La competencia digital de los estudiantes universitarios de primer curso de grado. Innoeduca. International Journal of Technology and Educational Innovation, 5(2), 104. https://doi.org/10.24310/innoeduca.2019.v5i2.5598

Túñez, M. y García, J. (2012). Las redes sociales como entorno docente: análisis del uso de Facebook en la docencia universitaria. Pixel Bit, Revista de Medios y Educación, 41, 77-92.

Web-Long, C. y Chun-Yi, L. (2013). Trust as a learning facilitator that affects students' learning performance in the Facebook community: An investigation in a business planning writing course. Computers y Education, $62,320-327$.

Julio Barroso-Osuna

Catedrático de la Universidad de Sevilla adscrito al Departamento de Didáctica y Organización Educativa de la Facultad de Ciencias de la Educación. Es miembro del Grupo de Investigación Didáctica (GID-HUM 390): Análisis Tecnológico y cualitativo. Ha participado en numerosas investigaciones y cursos relacionados con la temática de las Nuevas Tecnologías Aplicadas a la Educación. Su experiencia docente también está relacionada con el tópico mencionado. 


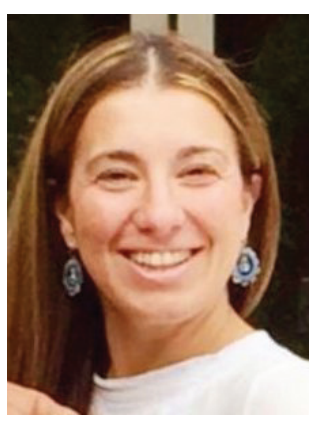

\section{Carmen Llorente-Cejudo}

Profesora Titular de la Universidad de Sevilla adscrita al Departamento de Didáctica y Organización Educativa de la Facultad de Ciencias de la Educación. Es miembro del Grupo de Investigación Didáctica (GID-HUM 390): Análisis Tecnológico y cualitativo. Ha participado en numerosas publicaciones nacionales e internacionales relacionados con la Tecnología Educativa. Su experiencia docente también está relacionada con el tópico mencionado.

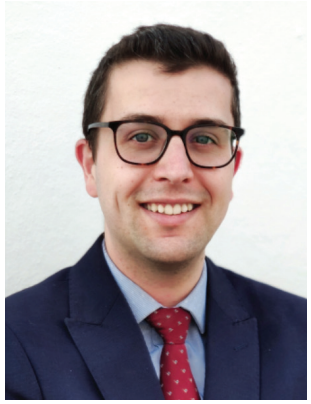

\section{Antonio Palacios-Rodríguez}

Graduado en Educación Primaria por la Universidad de Sevilla. También ha realizado el Máster Universitario en Dirección, Evaluación y Calidad de las Instituciones de Formación. Actualmente trabaja como Contratado Predoctoral FPU en el Departamento de Didáctica y Organización Educativa de la Facultad de Ciencias de la Educación de la Universidad de Sevilla. Su experiencia docente e investigadora está relacionada con la Tecnología Educativa. 